\title{
Soft-collinear effective theory: BRST formulation
}

\author{
Sudhaker Upadhyay ${ }^{1,2,3, a}$, Bhabani Prasad Mandal ${ }^{4, b}$ \\ ${ }^{1}$ Department of Physics, K.L.S. College (a constituent unit of Magadh University, Bodh-Gaya), Nawada, Bihar 805110, India \\ ${ }^{2}$ Visiting Associate, Inter-University Centre for Astronomy and Astrophysics (IUCAA), Pune, Maharashtra 411007, India \\ ${ }^{3}$ School of Physics, Damghan University, Damghan 3671641167, Iran \\ ${ }^{4}$ Department of Physics, Institute of Science, Banaras Hindu University, Varanasi 221005, India
}

Received: 17 October 2020 / Accepted: 8 March 2021 / Published online: 2 April 2021

(C) The Author(s) 2021

\begin{abstract}
We provide a BRST formalism for the softcollinear effective theory describing interactions of soft and collinear degrees of freedom in the presence of a hard interaction. In particular, we develop a BRST symmetry transformation for SCET theory. We further generalize the BRST formulation by making the transformation parameter field dependent. This establishes a mapping between several SCET actions consistently when defined in different gauge conditions. In fact, a definite structure of gauge-fixed actions corresponding to any particular gauge condition can be generated for SCET theory using our formulation.
\end{abstract}

\section{Contents}

1 Introduction . . . . . . . . . . . . . 1

2 SCET I action and BRST symmetry . . . . . . . . . . 2

3 The FDBRST transformation . . . . . . . . . . 4

3.1 General setup . . . . . . . . . . . . 4

3.2 Collinear FDBRST transformation . . . . . . . 4

3.3 Ultrasoft FDBRST transformation . . . . . . . 4

3.4 Implementation of FDBRST transformation . . 5

4 Applications and conclusions ......... . 5 5

References . . . . . . . . . . . . . . 6

\section{Introduction}

Effective field theories are used to separate the contributions associated with different scales, in particular, a highenergy and a low-energy scales of quantum chromodynamics (QCD). Over the past two decades, soft-collinear effective field theory (SCET) [1-4] has become amongst one of the

\footnotetext{
a e-mails:

sudhakerupadhyay@gmail.com;

haker@associates.iucaa.in

b e-mail: bhabani.mandal@ gmail.com (corresponding author)
}

important theories describing low-energy effective field theories of the Standard Model. In QCD, the low-energy part is nonperturbative in particular. In order to derive the factorization theorems and to perform the resummation of Sudakov logarithms, SCET provides an alternative to the traditional diagrammatic techniques [5]. SCET has been applied to a large variety of processes, from $B$-meson decays to jet production at the Large Hadron Collider (LHC). In Ref. [4], the factorization of soft and ultrasoft gluons from collinear particles is shown at the level of operators.

In order to describe jet-like events of QCD in SCET, it is convenient to write fields in either collinear, anti-collinear or soft (low energetic) modes with the help of the light cone unit vectors satisfying $n^{2}=\bar{n}^{2}=0$ and $n \cdot \bar{n}=2$. A momentum in the light-cone basis is represented as

$$
\begin{aligned}
p^{\mu} & =\frac{n^{\mu}}{2} \bar{n} \cdot p+\frac{\bar{n}^{\mu}}{2} n \cdot p+p_{\perp}^{\mu}, \\
& =\frac{n^{\mu}}{2} p^{-}+\frac{\bar{n}^{\mu}}{2} p^{+}+p_{\perp}^{\mu},
\end{aligned}
$$

where $\perp$ components are orthogonal to both collinear unit vector $n$ and anti-collinear unit vector $\bar{n}$.

The gauge symmetry structure in SCET is richer than the QCD as the former involves more than one distinct gluon fields. Therefore, the idea of background fields is required to give well defined meaning to several distinct gluon fields [6]. Based on momentum regions, SCET is categorized in two formulations: SCET I and SCET II. SCET I and SCET II scale soft sector of the theory differently. For instance, in SCET I all the momentum components of the soft fields are scaled similar to the small component of the collinear fields, while in SCET-II the momentum components of soft fields are scaled like the transverse component of the collinear fields.

Becchi-Rouet-Stora-Tyutin (BRST) formulation is a comparatively rigorous mathematical scheme [7-9] which provides a powerful technique to quantise gauge field the- 
ories. The range of applicability of BRST formulation further enhanced by extending it, where the anti-commuting transformation parameter is made finite and field-dependent [10]. The finite field-dependent BRST transformations have been studied for various field theoretic systems with gauge symmetries and have been found many applications [11-43]. Although BRST formulation simplifies the renormalization procedure greatly and helps to show unitarity of many theories, the implementation of this approach in SCET is quite cumbersome task. Thus even though a full field theoretic description for hadronic processes is developed, the BRST formulation for SCET is not studied so far. This provides us with an opportunity to bridge this gap.

In this paper, we consider a gauge invariant SCET I action which admits different sets of gauge invariance in different momentum regions. We develop two sets of BRST symmetries which leave the Faddeev-Popov effective actions for collinear and ultrasoft sectors invariant, separately. Moreover, we formulate an generalized version of BRST symmetries by making the transformation parameter field dependent. We call such transformation field-dependent BRST (FDBRST) transformation. In contrast to the standard case, this eventually leads to a non-trivial Jacobian for functional measure in the expression of transition amplitude. This Jacobian extends the BRST-exact parts of the action. We show that for some appropriate choices of field-dependent parameters, an exact form of gauge-fixed action corresponding to different gauge conditions can be generated through FDBRST.

The plan of the paper is as following. In Sect. 2, we construct fermionic rigid collinear and ultrasoft BRST transformations. These symmetry transformations are further generalized by making the transformation parameters field dependent in a traditional way in Sect. 3. Moreover, we implement such FDBRST transformations with appropriately constructed transformation parameters to the generating functional. We summarize the outcome of this formulation with their significance in the Sect. 4.

\section{SCET I action and BRST symmetry}

In the Refs. $[4,44]$, it has been shown that the leading-power SCET collinear quark action in the expansion in the SCET expansion parameter should satisfy following requirements: (a) it should yield proper spin structure of the collinear propagator, (b) it should have both collinear quarks and collinear antiquarks, (c) it should interact with both collinear gluons and ultrasoft gluons, (d) and it should lead to the correct loworder propagator for different situations. These requirements allow us to write down the effective leading-power SCET action. Further by splitting the fermion field into big and small components using the usual projectors $\left(\frac{h i \hbar}{4}\right.$ and $\frac{\hbar h h}{4}$ ) and eliminating (using the equations of motion) the small components, one can write first the leading-power collinear quark action with collinear modes in $n$ direction as [4]

$$
\begin{aligned}
S_{n \xi}= & \int d^{4} x\left[e^{x \cdot \mathcal{P}} \bar{\xi}_{n}(i n \cdot D\right. \\
& \left.\left.+i \not D_{n \perp} \frac{1}{i \bar{n} \cdot D_{n}} i \not D_{n \perp}\right) \frac{\not \hbar}{2} \xi_{n}\right],
\end{aligned}
$$

where $\mathcal{P}^{\mu}$ is a label operator which provides a definite power counting for derivatives. Now to separate momentum scales, we define our momentum $p^{\mu}$ to be a sum of a large momentum components $p_{l}^{\mu}$ called the label momentum and a small momentum $p_{r}^{\mu}$ called the residual momentum. The label operator extracts the momentum label of a given field as follows

$\mathcal{P}^{\mu} \xi_{n, p_{l}}(x)=p_{l}^{\mu} \xi_{n, p_{l}}(x)$

Here we note that, in operator products, $n \cdot \mathcal{P}$ acts on the anticollinear fields and $\bar{n} \cdot \mathcal{P}$ acts on the collinear fields only. The collinear covariant derivatives are defined as

$$
\begin{aligned}
i n \cdot D & =i n \cdot \partial+g n \cdot A_{n}+g n \cdot A_{u s}, \\
i \not D_{n \perp}^{\mu} & =\mathcal{P}_{\perp}^{\mu}+g A_{n \perp}^{\mu}, \\
i \bar{n} \cdot D_{n} & =\overline{\mathcal{P}}+g \bar{n} \cdot A_{n} .
\end{aligned}
$$

Even in the presence of ultrasoft fields, one can write collinear quark action equivalent to (2.1) as

$$
S_{n \xi}=\int d^{4} x\left[e^{x \cdot \mathcal{P}} \bar{\Xi}_{n} i \not D \Xi_{n}\right], \quad \Xi_{n} \equiv\left(\begin{array}{c}
\xi_{n} \\
\varphi_{\bar{n}}
\end{array}\right)
$$

where spinor components $\varphi_{\bar{n}}$ are subleading in the collinear limit and

$i \not D=\frac{\hbar \hbar}{2} i n \cdot D+\frac{\not h}{2} i \bar{n} \cdot D_{n}+i \not D_{n \perp}=i \not D_{n}+\frac{\not \hbar}{2} g n \cdot A_{u s}$.

In order to write the collinear gluon action, ultrasoft gauge field $A_{u s}^{\mu}$ is treated as a background field with respect to collinear gauge field $A_{\mu}^{n}$. In this way, the QCD gluon action leads to the leading-power collinear gluon action in a covariant gauge as follows [4]

$$
\begin{aligned}
S_{n g}= & \int d^{4} x \operatorname{Tr}\left[\frac{1}{2 g^{2}}\left(\left[i \mathcal{D}^{\mu}, i \mathcal{D}_{\mu}\right]\right)^{2}+\tau\left(\left[i \mathcal{D}_{u s}^{\mu}, A_{n \mu}\right]\right)^{2}\right. \\
& \left.+2 \bar{c}_{n}\left[i \mathcal{D}_{u s}^{\mu},\left[i \mathcal{D}_{\mu}, c_{n}\right]\right]\right]
\end{aligned}
$$


where $\tau$ is a gauge fixing parameter for collinear gluon and

$$
\begin{aligned}
i \mathcal{D}^{\mu}= & \frac{n^{\mu}}{2}\left(\overline{\mathcal{P}}+g \bar{n} \cdot A_{n}\right)+\left(\mathcal{P}_{\perp}^{\mu}+g A_{\perp, n}^{\mu}\right) \\
& +\frac{\bar{n}^{\mu}}{2}\left(i n \cdot \partial+g n \cdot A_{n}+g n \cdot A_{u s}\right), \\
i \mathcal{D}_{u s}^{\mu}= & \frac{n^{\mu}}{2} \overline{\mathcal{P}}+\mathcal{P}_{\perp}^{\mu}+\frac{\bar{n}^{\mu}}{2}\left(i n \cdot \partial+g n \cdot A_{u s}\right) .
\end{aligned}
$$

The leading-power Faddeev-Popov action for ultrasoft quarks and ultrasoft gluons in a covariant gauge can be written [4] as

$$
\begin{aligned}
S_{u s}= & \int d^{4} x\left[\bar{\psi}_{u s} i D_{u s} \psi_{u s}-\operatorname{Tr}\left(\frac{1}{2} G_{\mu \nu}^{u s} G_{u s}^{\mu \nu}\right.\right. \\
& \left.\left.+\tau_{u s}\left(\partial_{\mu} A_{u s}^{\mu}\right)^{2}+2 \bar{c}_{u s} \partial_{\mu} D_{u s}^{\mu} c_{u s}\right)\right],
\end{aligned}
$$

where $\tau_{u s}$ is a gauge fixing parameter for ultrasoft gluon and $i D_{u s}^{\mu}=i \partial^{\mu}+A_{u s}^{\mu}$. Here we note that the forms of subleading Lagrangian are also presented in Ref. [45] but we are concerned with the leading power Lagrangian only as the number of terms in the sub-leading Lagrangian which actually contribute is relatively small since most terms involve higher numbers of fields.

The complete Faddeev-Popov effective action for a single set of quark and gluon collinear modes in the $n$ direction, and quark and gluon ultrasoft modes in a covariant gauge is given by

$S_{\text {scet }}=S_{n \xi}+S_{n g}+S_{u s}$.

Here we must comment that for processes with multiple energetic hadron, or multiple energetic jet our list of degrees of freedom must include multiple collinear modes, and therefore multiple collinear quark and collinear gluon. After interaction of two collinear modes in different directions, the resulting particle does not change the formulation of the leading order collinear Lagrangians.

The kinetic part of the SCET action admits two types of gauge transformations; the collinear gauge transformation

$U_{c}=e^{i \alpha_{c}^{a}(x) T^{a}}$,

and the ultrasoft gauge transformation

$U_{u s}=e^{i \alpha_{u s}^{a}(x) T^{a}}$.

Under infinitesimal collinear gauge transformation collinear fields transform as

$$
\begin{aligned}
\xi_{n} & \rightarrow \xi_{n}+i \alpha_{n} \xi_{n}, \\
A_{n}^{\mu} & \rightarrow A_{n}^{\mu}+\left[i \mathcal{D}^{\mu}, \alpha_{n}\right],
\end{aligned}
$$

and under infinitesimal ultrasoft gauge transformation ultrasoft fields transform as

$$
\begin{aligned}
\psi_{u s} & \rightarrow \psi_{u s}+i \alpha_{u s} \psi_{u s}, \\
A_{u s}^{\mu} & \rightarrow A_{u s}^{\mu}+i D_{u s}^{\mu} \alpha_{u s} .
\end{aligned}
$$

Plugging $\alpha_{n}=c_{n}$ in (2.12), with $c_{n}$ being a local Grassmann field, we obtain

$$
\begin{aligned}
s_{b} \xi_{n} & =i c_{n} \xi_{n}, \\
s_{b} A_{n}^{\mu} & =\left[i \mathcal{D}^{\mu}, c_{n}\right] .
\end{aligned}
$$

Under such a transformation, $S_{n \xi}$ and kinetic part of $S_{n g}$ remain invariant as the ghost field does not affect the original gauge invariance. From differential geometry in the case of an infinite-dimensional Lie group together with its Lie algebra one can define a Maurer-Cartan differential form $\omega$, which is a left-invariant 1-form. It is well-known that such a Maurer-Cartan form $\omega$ satisfies $s_{b} \omega=-\frac{1}{2} \omega \times \omega$. By considering $\omega(x)=-g c_{n}(x)$, we write the following "on-shell nilpotent" BRST transformations:

$s_{b} c_{n}=\frac{g}{2} c_{n} c_{n}$,

$s_{b} \bar{c}_{n}=\tau\left[i \mathcal{D}_{u s}^{\mu}, A_{n \mu}\right]$.

Here, on-shell nilpotency signifies that the transformation is nilpotent (i.e. $s_{b}^{2}=0$ ) only when one utilizes the equation of motion for the field $\bar{c}_{n}$. We would like to add that above BRST transformation can be made off shell nilpotent by introducing Nakanishi-Lautrup (NL) [46] type auxiliary fields. The operator $s_{b}$ refers to the Slavnov variation which characterizes BRST transformation without a transformation parameter. In the present formulation Slavnov variation is onshell nilpotent in the sector of anti-ghost field. However this Slavnov variation can be made off-shell nilpotent by introducing the suitable NL type auxiliary fields in the background field method in order to linearize the gauge-fixing terms of the Faddeev-Popov action.

Now, we write the above on-shell nilpotent collinear BRST transformation in terms of collinear transformation parameter $\Lambda_{n}$ as

$$
\begin{aligned}
\delta_{b} \xi_{n} & =i c_{n} \xi_{n} \Lambda_{n}, \\
\delta_{b} \bar{\xi}_{n} & =-i \bar{\xi}_{n} c_{n} \Lambda_{n}, \\
\delta_{b} A_{n}^{\mu} & =\left[i \mathcal{D}^{\mu}, c_{n}\right] \Lambda_{n}, \\
\delta_{b} c_{n} & =\frac{g}{2} c_{n} c_{n} \Lambda_{n}, \\
\delta_{b} \bar{c}_{n} & =\tau\left[i \mathcal{D}_{u s}^{\mu}, A_{n \mu}\right] \Lambda_{n} .
\end{aligned}
$$

In the same fashion, we write on-shell nilpotent ultrasoft BRST transformation in terms of ultrasoft transformation parameter $\Lambda_{u s}$ as

$$
\begin{aligned}
\delta_{b} \psi_{u s} & =i c_{u s} \psi_{u s} \Lambda_{u s}, \\
\delta_{b} \bar{\psi}_{u s} & =-i \bar{\psi}_{u s} c_{u s} \Lambda_{u s}, \\
\delta_{b} A_{u s}^{\mu} & =i D_{u s}^{\mu} c_{u s} \Lambda_{u s}, \\
\delta_{b} c_{u s} & =\frac{g}{2} c_{u s} c_{u s} \Lambda_{u s}, \\
\delta_{b} \bar{c}_{u s} & =\tau_{u s} i \partial^{\mu} A_{u s}^{\mu} \Lambda_{u s} .
\end{aligned}
$$


The effective action in Eq. (2.9) remains invariant under these transformations. These sets of BRST symmetries are very important in order to renormalize the Feynman diagrams. With the help of these BRST transformations one can write Slavnov-Taylor identities for generating function. The transformation parameters $\Lambda_{n}$ and $\Lambda_{u s}$ are infinitesimal anticommuting parameters. The generating functional for SCET action can be written as

$Z[0]=\int\left[D \phi_{n}\right]\left[D \phi_{u s}\right] \exp \left(i S_{\text {scet }}\right)$,

where $\phi_{n}$ and $\phi_{u s}$ are generic notations for collective collinear and ultrasoft fields respectively. It is straightforward to check the invariance of collinear and ultrasoft path integral measures under BRST symmetry transformations as the Jacobian calculation gives unit.

\section{The FDBRST transformation}

\subsection{General setup}

To construct FDBRST we use a generic notation for the BRST transformations in Eqs. (2.16) and (2.17) for a collective field (having both collinear and ultrasoft fields) $\phi_{n(u s)}(x)$ as follows:

$\delta_{b} \phi_{n(u s)}(x)=s_{b} \phi_{n(u s)}(x) \Lambda_{n(u s)}$.

Here we note that the nature of nilpotency, being on-shell or off-shell, do not affect the general setup of FDBRST transformation [10]

Following the standard procedure [10], a field-dependent BRST transformation is constructed via interpolation of a continuous parameter $\kappa(0 \leq \kappa \leq 1)$ as:

$\frac{d \phi_{n(u s)}(x, \kappa)}{d \kappa}=s_{b} \phi_{n(u s)}(x, \kappa) \Theta_{n(u s)}^{\prime}\left[\phi_{n(u s)}(\kappa)\right]$,

where $\Theta_{n(u s)}^{\prime}\left[\phi_{n(u s)}(\kappa)\right]$ is an infinitesimal field-dependent parameter. In contrast to standard BRST transformation, this field-dependent transformation is not the symmetry of the path integral measure and amounts a precise Jacobian in the generating functional. This Jacobian contribution can be expressed as exponential of some functional of local fields and modifies the BRST exact part of the action [10]. The Jacobian of functional measure is given by $[47,48]$

$$
\begin{aligned}
& J\left[\phi_{n(u s)}\right] \\
& =\exp \left(-\int d^{4} x \sum_{\phi_{n(u s)}} \pm s_{b} \phi_{n(u s)}(x) \frac{\delta \Theta_{n(u s)}^{\prime}\left[\phi_{n(u s)}(x)\right]}{\delta \phi_{n(u s)}(x)}\right) .
\end{aligned}
$$

This Jacobian therefore extrapolates the action (within functional integration) of the SCET theory (2.18) as follows:

$$
\begin{aligned}
Z[0] \longrightarrow & \int\left[D \phi_{n}\right]\left[D \phi_{u s}\right] \exp \left(i S_{\text {scet }}\right. \\
& \left.-\int d^{4} x \sum_{\phi_{n(u s)}} \pm s_{b} \phi_{n(u s)} \frac{\delta \Theta_{n(u s)}^{\prime}\left[\phi_{n(u s)}\right]}{\delta \phi_{n(u s)}}\right)
\end{aligned}
$$

This modified expression due to FDBRST does not amount any changes in the physical content of the theory but rather simplifies various issues in a dramatic way. In the next subsection we are going to demonstrate this.

\subsection{Collinear FDBRST transformation}

Following above methodology, which is applicable to both on-shell as well as off-shell BRST transformations, we construct the infinitesimal collinear FDBRST transformations as

$$
\begin{aligned}
\frac{d \xi_{n}}{d \kappa} & =i c_{n} \xi_{n} \Theta_{n}^{\prime}, \\
\frac{d \bar{\xi}_{n}}{d \kappa} & =-i \bar{\xi}_{n} c_{n} \Theta_{n}^{\prime}, \\
\frac{d A_{n}^{\mu}}{d \kappa} & =\left[i \mathcal{D}^{\mu}, c_{n}\right] \Theta_{n}^{\prime}, \\
\frac{d c_{n}}{d \kappa} & =\frac{g}{2} c_{n} c_{n} \Theta_{n}^{\prime}, \\
\frac{d \bar{c}_{n}}{d \kappa} & =\tau\left[i \mathcal{D}_{u s}^{\mu}, A_{n \mu}\right] \Theta_{n}^{\prime},
\end{aligned}
$$

where $\Theta_{n}^{\prime}$ is an infinitesimal collinear field-dependent transformation parameter. This parameter can be chosen arbitrarily provided that it must be nilpotent in nature. In the next section we will construct appropriate $\Theta_{n}^{\prime}$ to show how the generating functionals corresponding to various effective actions in different gauges are related.

\subsection{Ultrasoft FDBRST transformation}

In the similar fashion the infinitesimal ultrasoft FDBRST transformations are derived as

$$
\begin{aligned}
\frac{d \psi_{u s}}{d \kappa} & =i c_{u s} \psi_{u s} \Theta_{u s}^{\prime}, \\
\frac{d \bar{\psi}_{u s}}{d \kappa} & =-i \bar{\psi}_{u s} c_{u s} \Theta_{u s}^{\prime}, \\
\frac{d A_{u s}^{\mu}}{d \kappa} & =i D_{u s}^{\mu} c_{u s} \Theta_{u s}^{\prime}, \\
\frac{d c_{u s}}{d \kappa} & =\frac{g}{2} c_{u s} c_{u s} \Theta_{u s}^{\prime}, \\
\frac{d \bar{c}_{u s}}{d \kappa} & =\tau_{u s} i \partial^{\mu} A_{u s}^{\mu} \Theta_{u s}^{\prime},
\end{aligned}
$$


where $\Theta_{u s}^{\prime}$ is an arbitrary infinitesimal ultrasoft fielddependent transformation parameter.

\subsection{Implementation of FDBRST transformation}

In this subsection, we assign some specific values for the field dependent parameters $\Theta_{n}^{\prime}$ and $\Theta_{u s}^{\prime}$ and calculate Jacobians of the functional measures under respective FDBRST transformations. In this regard, we first choose the parameter of collinear FDBRST transformation $\Theta_{n}^{\prime}$ as

$$
\begin{aligned}
\Theta_{n}^{\prime}= & -i \int d^{4} y \operatorname{Tr}\left(\bar{c}_{n}\left[i \mathcal{D}_{u s}^{\mu}, A_{n \mu}\right]\right. \\
& \left.-\bar{c}_{n} f_{1}\left[A_{n \mu}, A_{u s}^{\mu}\right]\right),
\end{aligned}
$$

where $f_{1}\left[A_{n \mu}, A_{u s}^{\mu}\right]$ is the most general collinear gauge condition. For this parameter, the Jacobian of functional measure (3.3) yields with

$$
\begin{aligned}
S_{n g}^{f}= & \int d^{4} x \operatorname{Tr}\left[\frac{1}{2 g^{2}}\left(\left[i \mathcal{D}^{\mu}, i \mathcal{D}_{\mu}\right]\right)^{2}+\tau f_{1}^{2}\left[A_{n \mu}, A_{u s}^{\mu}\right]\right. \\
& \left.+2 \bar{c}_{n}\left[\frac{d f_{1}}{d A_{n \mu}},\left[i \mathcal{D}_{\mu}, c_{n}\right]\right]\right] \\
S_{u s}^{f}= & \int d^{4} x\left[\bar{\psi}_{u s} i \not D_{u s} \psi_{u s}-\operatorname{Tr}\left(\frac{1}{2} G_{\mu \nu}^{u s} G_{u s}^{\mu \nu}\right.\right. \\
& \left.\left.-\tau_{u s} f_{2}^{2}\left[A_{u s}^{\mu}\right]-2 \bar{c}_{n} \frac{d f_{2}}{d A_{u s}^{\mu}} i D_{u s}^{\mu} c_{u s}\right)\right]
\end{aligned}
$$

These are nothing but the leading-power collinear gluon action with gauge-fixing condition $f_{1}\left[A_{n \mu}, A_{u s}^{\mu}\right]=0$ and leading-power action for ultrasoft quarks and ultrasoft gluons with gauge-fixing condition $f_{2}\left[A_{u s}^{\mu}\right]=0$. Thus, FDBRST transformation upon implementation on generating functional changes the gauge-fixing and ghost sectors of collinear and ultrasoft gluon actions. This result will be very useful in handling the Feynman processes of the theory. Since calculations of different Green's functions depend on the choice of

$$
J_{n}=e^{-i \int d^{4} x \operatorname{Tr}\left[\tau\left(\left[i \mathcal{D}_{u s}^{\mu}, A_{n \mu}\right]\right)^{2}+2 \bar{c}_{n}\left[i \mathcal{D}_{u s}^{\mu},\left[i \mathcal{D}_{\mu}, c_{n}\right]\right]-f_{1}^{2}\left[A_{n \mu}, A_{u s}^{\mu}\right]-2 \bar{c}_{n}\left[\frac{d f_{1}}{d A_{n \mu}},\left[i \mathcal{D}_{\mu}, c_{n}\right]\right]\right]} .
$$

Here, we try to emphasize that we utilize an appropriate BRST transformation for the antighost fields according to gauge conditions. On the other hand the field dependent parameter $\Theta_{u s}^{\prime}$ for ultrasoft BRST is chosen as

$\Theta_{u s}^{\prime}=-i \int d^{4} y \operatorname{Tr}\left(\bar{c}_{n}\left(i \partial_{\mu} A_{u s}^{\mu}\right)-\bar{c}_{n} f_{2}\left[A_{u s}^{\mu}\right]\right)$

The Jacobian of functional measure (3.3) with this parameter leads to

$J_{u s}=e^{i \int d^{4} x \operatorname{Tr}\left[\tau_{u s}\left(\partial_{\mu} A_{u s}^{\mu}\right)^{2}+2 \bar{c}_{u s} \partial_{\mu} D_{u s}^{\mu} c_{u s}+f_{2}^{2}\left[A_{u s}^{\mu}\right]+2 \bar{c}_{n} \frac{d f_{2}}{d A_{u s}^{\mu}} i D_{u s}^{\mu} c_{u s}\right]}$.

These results eventually imply that under FDBRST transformation with the above field dependent parameters

$$
\begin{aligned}
Z[0] & =\int\left[D \phi_{n}\right]\left[D \phi_{u s}\right] \exp \left(i S_{\text {scet }}\right) \\
& \longrightarrow \int\left[D \phi_{n}\right]\left[D \phi_{u s}\right] \exp \left(i S_{\text {scet }}^{f}\right),
\end{aligned}
$$

where final effective action is defined by

$$
S_{\text {scet }}^{f}=S_{n \xi}+S_{n g}^{f}+S_{u s}^{f}
$$

gauge-fixing condition and for some particular choices the calculations are simplified greatly. For instance, the gluon jet function at two-loop order is computed in both Feynman and light-cone gauge and has found that the latter leads to considerable simplifications [49]. But our result is not limited to a particular set of gauges but holds for any arbitrary sets of gauges. According to the standard procedure, Green's functions in initial gauge under the FDBRST transformation transform as

$$
\begin{aligned}
\left\langle\left\langle G^{f}\right\rangle\right\rangle= & \int\left[D \phi_{n}^{\prime}\right]\left[D \phi_{u s}^{\prime}\right] \mathcal{O}\left[\phi_{n(u s)}^{\prime}\right] \times \exp \left[i S_{\mathrm{scet}}^{f}\left[\phi_{n(u s)}^{\prime}\right]\right] \\
= & \int\left[D \phi_{n}\right]\left[D \phi_{u s}\right]\left(\phi_{n(u s)}\right. \\
& \left.+s_{b} \phi_{n(u s)} \Theta_{n(u s)}\right) \exp \left[i S_{\text {scet }}\right] \\
= & \langle\langle G\rangle\rangle+\langle\langle\Delta G\rangle\rangle
\end{aligned}
$$

where $\mathcal{O}\left[\phi_{n(u s)}^{\prime}\right]$ is an arbitrary operator and $\Delta G$ is difference between the Green's functions in the two sets of gauges. The structure of Faddeev-Popov action corresponding to that gauge can be achieved easily from this FDBRST formulation.

\section{Applications and conclusions}

In this paper, we have considered an effective theory, in the light-cone coordinates which describes the interactions of soft and collinear modes in the presence of a hard interaction. By eliminating the small components after decompo- 
sition of fermion field, we have written a gauge invariant SCET I action which admits different sets of gauge invariance in the different momentum regions. In order to quantize correctly, we need to extend the classical action by adding suitable terms which break the local gauge invariance. Such gauge variant terms attribute ghost terms in the generating functional of the theory. We have developed two independent sets of on-shell nilpotent BRST symmetries which leave the Faddeev-Popov actions for collinear and ultrasoft sectors invariant separately. The nilpotency of order two can be proved only utilizing equation of motion of corresponding anti-ghost field. These BRST transformations may help to write the counter terms to make the theory renormalizable.

Furthermore, we have generalized these sets of BRST symmetries by making the transformation parameter field dependent. The difference of these generalized symmetries to the usual one lies to the fact that these are not the symmetries of the functional measure and, in contrast to the usual one, eventually lead to a local Jacobian. On the physical ground, this Jacobian do not modify the theory as all the changes attributed to the BRST-exact parts of the action. We have shown that for some specific choices of field-dependent parameters the exact expressions for various gauge-fixed actions can suitably be derived. These results are of particular importance for the theoretical estimation of decay processes. Because of this certain diagram calculations get simplified greatly in some particular gauge choices. For instance, it has been shown that by extending SCET formulation to the class of singular gauges, a new Wilson line, the $T$ Wilson line, has to be invoked as a basic SCET building block [50,51]. It is shown there that study in non-covariant gauges extend the range of applicability of SCET. The transition from one gauge to another in SCET can easily be done through our approach. Here we remark that the formulation of soft-collinear effective theory has been already been worked out to second order in a power series $[1,2,52]$. It would be interesting to develop the complete BRST description for higher order in a power series which is a subject of future investigation.

Data Availability Statement This manuscript has no associated data or the data will not be deposited. [Authors' comment: The paper is analytical and calculated manually. The paper does not contains any of the data.]

Open Access This article is licensed under a Creative Commons Attribution 4.0 International License, which permits use, sharing, adaptation, distribution and reproduction in any medium or format, as long as you give appropriate credit to the original author(s) and the source, provide a link to the Creative Commons licence, and indicate if changes were made. The images or other third party material in this article are included in the article's Creative Commons licence, unless indicated otherwise in a credit line to the material. If material is not included in the article's Creative Commons licence and your intended use is not permitted by statutory regulation or exceeds the permitted use, you will need to obtain permission directly from the copyright holder. To view a copy of this licence, visit http://creativecomm ons.org/licenses/by/4.0/.

Funded by SCOAP ${ }^{3}$.

\section{References}

1. C.W. Bauer, S. Fleming, D. Pirjol, I.W. Stewart, Phys. Rev. D 63, $114020(2001)$

2. M. Beneke, A.P. Chapovsky, M. Diehl, T. Feldmann, Nucl. Phys. B 643, 431 (2002)

3. C.W. Bauer, I.W. Stewart, Phys. Lett. B 516, 134 (2001)

4. C.W. Bauer, D. Pirjol, I.W. Stewart, Phys. Rev. D 65, 054022 (2002)

5. J. Collins, Foundations of Perturbative QCD (Cambridge University Press, Cambridge, 2011)

6. L.F. Abbott, Nucl. Phys. B 185, 189 (1981)

7. C. Becchi, A. Rouet, R. Stora, Ann. Phys. 98, 287 (1974)

8. I.V. Tyutin, LEBEDEV 75, 39 (1975)

9. S. Weinberg, The Quantum Theory of Fields, Vol-II: Modern Applications (University Press, Cambridge, 1996)

10. S.D. Joglekar, B.P. Mandal, Phys. Rev. D 51, 1919 (1995)

11. S.D. Joglekar, B.P. Mandal, Int. J. Mod. Phys. A 17, 1279 (2002)

12. S. Upadhyay, S.K. Rai, B.P. Mandal, J. Math. Phys. 52, 022301 (2011)

13. S. Upadhyay, B.P. Mandal, Eur. Phys. J. C 75, 327 (2015)

14. S. Upadhyay, B.P. Mandal, Int. J. Theor. Phys. 55, 1 (2016)

15. S. Upadhyay, B.P. Mandal, Phys. Lett. B 744, 231 (2015)

16. S. Upadhyay, B. P. Mandal, Prog. Theor. Exp. Phys. 053B04, 1 (2014)

17. S. Upadhyay, B.P. Mandal, Eur. Phys. J. C 72, 2065 (2012)

18. S. Upadhyay, B.P. Mandal, Ann. Phys. 327, 2885 (2012)

19. S. Upadhyay, B.P. Mandal, Eur. Phys. Lett. 93, 31001 (2011)

20. S. Upadhyay, B.P. Mandal, Mod. Phys. Lett. A 25, 3347 (2010)

21. M. Faizal, B.P. Mandal, S. Upadhyay, Phys. Lett. B 721, 159 (2013)

22. M. Faizal, S. Upadhyay, B.P. Mandal, Eur. Phys. J. C 76, 189 (2016)

23. M. Faizal, S. Upadhyay, B.P. Mandal, Int. J. Mod. Phys. A 30, $1550032(2015)$

24. M. Faizal, S. Upadhyay, B.P. Mandal, Phys. Lett. B 738, 201 (2014)

25. S. Upadhyay, D. Das, Phys. Lett. B 733, 63 (2014)

26. S. Upadhyay, M. Faizal, P.A. Ganai, Int. J. Mod. Phys. A 30, 1550185 (2015)

27. S. Upadhyay, B. Paul, Eur. Phys. J. C 76, 394 (2016)

28. R. Banerjee, S. Upadhyay, Phys. Lett. B 734, 369 (2014)

29. S. Upadhyay, P. A. Ganai, Prog. Theor. Exp. Phys. 063B04, 1 (2016)

30. B.P. Mandal, S.K. Rai, S. Upadhyay, EPL 92, 21001 (2010)

31. S. Upadhyay, A. Reshetnyak, B.P. Mandal, Eur. Phys. J. C 76, 391 (2016)

32. R. Banerjee, B. Paul, S. Upadhyay, Phys. Rev. D 88, 065019 (2013)

33. P.Y. Moshin, A.A. Reshetnyak, Nucl. Phys. B 888, 92 (2014)

34. P.Y. Moshin, A.A. Reshetnyak, Int. J. Mod. Phys. A 30, 1550021 (2015)

35. P.Y. Moshin, A.A. Reshetnyak, Phys. Lett. B 739, 110 (2014)

36. S. Upadhyay, Phys. Lett. B 740, 341 (2015)

37. S. Upadhyay, Ann. Phys. 356, 299 (2015)

38. S. Upadhyay, Mod. Phys. Lett. A 30, 1550072 (2015)

39. S. Upadhyay, Ann. Phys. 340, 110 (2014)

40. S. Upadhyay, Ann. Phys. 344, 290 (2014)

41. S. Upadhyay, EPL 105, 21001 (2014)

42. S. Upadhyay, EPL 104, 61001 (2013)

43. S. Upadhyay, Phys. Lett. B 727, 293 (2013)

44. C.W. Bauer, I.W. Stewart, Notes on the soft-collinear effective theory. TASI lecture notes (2013)

45. I. Moult, I.W. Stewart, G. Vita, arXiv:1905.07411

46. N. Nakanishi, I. Ojima, Covariant Operator Formalism of Gauge Theory and Quantum Gravity (World Scientific, Singapore, 1990) 
47. PYu. Moshin, S. Upadhyay, R.A. Castro, Braz. J. Phys. 47, 411 (2017)

48. S. Upadhyay, M. Oksanen, R. Bufalo, Braz. J. Phys. 47, 350 (2017)

49. T. Becher, G. Bell, Phys. Lett. B 695, 252 (2011)
50. A. Idilbi, I. Scimemi, Phys. Lett. B 695, 463 (2011)

51. M. Garcia-Echevarria, A. Idilbi, I. Scimemi, Phys. Rev. D 84, 011502 (2011)

52. M. Beneke, T. Feldmann, Phys. Lett. B 553, 267 (2003) 